\title{
Modeling the Evolutionary Dynamics of Plasmids in Spatial Populations
}

\author{
Brian D. Connelly, Luis Zaman, Philip K. McKinley, and Charles Ofria \\ BEACON Center for the Study of Evolution in Action \\ Michigan State University \\ East Lansing, MI 48824, USA \\ \{bdc,zamanlui,mckinle3,ofria\}@msu.edu
}

\begin{abstract}
One of the processes by which microorganisms are able to rapidly adapt to changing conditions is horizontal gene transfer, whereby an organism incorporates additional genetic material from sources other than its parent. These genetic elements may encode a wide variety of beneficial traits. Under certain conditions, many computational models capture the evolutionary dynamics of adaptive behaviors such as toxin production, quorum sensing, and biofilm formation, and have even provided new insights into otherwise unknown or misunderstood phenomena. However, such models rarely incorporate horizontal gene transfer, so they may be incapable of fully representing the vast repertoire of behaviors exhibited by natural populations. Although models of horizontal gene transfer exist, they rarely account for the spatial structure of populations, which is often critical to adaptive behaviors.

In this work we develop a spatial model to examine how conjugation, one mechanism of horizontal gene transfer, can be maintained in populations. We investigate how both the costs of transfer and the benefits conferred affect evolutionary outcomes. Further, we examine how rates of transmission evolve, allowing this system to adapt to different environments. Through spatial models such as these, we can gain a greater understanding of the conditions under which horizontally-acquired behaviors are evolved and are maintained.
\end{abstract}

\section{Categories and Subject Descriptors}

H.4 [Information Systems Applications]: Miscellaneous; F.1.1 [Computation by Abstract Devices]: Models of Computation-Self-modifying machines

\section{General Terms}

Experimentation, Theory

\section{Keywords}

Artificial life, plasmid, horizontal gene transfer, antibiotic resistance, digital evolution

Permission to make digital or hard copies of all or part of this work for personal or classroom use is granted without fee provided that copies are not made or distributed for profit or commercial advantage and that copies bear this notice and the full citation on the first page. To copy otherwise, to republish, to post on servers or to redistribute to lists, requires prior specific permission and/or a fee.

GECCO'11, July 12-16, 2011, Dublin, Ireland.

Copyright 2011 ACM 978-1-4503-0557-0/11/07 ...\$10.00.

\section{INTRODUCTION}

Unlike more complex organisms, the exchange of genetic material is not limited in microorganisms to the process of replication. In addition to vertical transmission, which occurs from parent to offspring during replication, microorganisms are able to transfer genes horizontally. Horizontal gene transfer (HGT) enables organisms to incorporate new genetic material from mobile genetic elements during their lifetime, allowing them to rapidly adapt to ever-changing environmental conditions. It is becoming increasingly apparent that HGT plays a major role in the lives and evolution of bacteria [12] and, in fact, much of life, as even complex organisms trace their ancestry back in some way to singlecelled life [4]. Although these mobile genetic elements often provide advantages, they are also costly. Understanding the conditions under which the process of horizontal gene transfer can evolve and be maintained is critical to understanding bacterial life and how this process can be harnessed in artificial systems.

In this paper, we describe a computational model used to explore the relationship between selection for plasmidencoded adaptations and the costs of plasmid carriage in spatial populations. First, we examine how the abundance of a plasmid that provides antibiotic resistance differs in environments with different levels of antibiotic, and therefore provides different benefit. We hypothesize that resistanceconferring plasmids will be more abundant in environments with higher levels of antibiotic, as selection for this trait will be stronger.

Second, we examine how the cost associated with plasmid transmission affects their abundance in these environments. We accomplish this goal by modeling the rate at which transfer occurs as an evolved trait in plasmids. Since high transfer rates incur a high cost on hosts, plasmids must optimize their rate of transfer based on the potential benefits.

Two alternative hypotheses exist for how populations will react to evolving transfer rates in different environments. One possibility is that plasmids will adjust their transfer rate, and therefore cost, to match the benefit provided in different environments. Here, plasmids that confer greater benefit will evolve higher levels of transmission, while plasmids that confer less benefit will evolve lower rates of transmissions to compensate. Alternatively, all plasmids may simply maximize their transmission rates in order to proliferate, regardless of the detrimental effects on their hosts. Using the model presented in this work, we are able to exclude one of these competing hypotheses.

This work is presented as follows: Section 2 reviews the 
process of horizontal gene transfer via conjugation and discusses previous models of adaptive behaviors and HGT. Our model of horizontal gene transfer in spatial populations is introduced in Section 3, and experimental results are presented in Section 4. Finally, we interpret these results and look toward follow-up studies in Section 5.

\section{BACKGROUND AND RELATED WORK}

Plasmids are one type of mobile genetic element that abound in bacterial populations. These small DNA molecules encode a wide variety of beneficial traits such as antibiotic resistance, toxin production, and support for various metabolic processes, among many others [18]. Plasmids are often categorized according to their ability to transfer to other bacteria. Conjugative plasmids carry transfer genes, which encode processes necessary for transfer such as pilus production and mating pair formation, further described below. Mobilizable plasmids do not contain these genes, and therefore require other means of transfer. This generally involves exploiting the transfer mechanisms of another plasmid in the host. Although plasmids often confer beneficial adaptations, their carriage and transfer are also costly. These costs can be attributed to the synthesis of plasmid DNA and the expression of genes related to plasmid-encoded traits.

One common mechanism for the horizontal transfer of plasmids is conjugation, a process in which a bacterial donor and recipient establish direct cell-cell contact and exchange plasmids, illustrated in Figure 1. During this process, the donor cell produces a pilus, which allows it to connect to the recipient cell. A single strand of the double-stranded DNA is then passed to the recipient. Subsequently, both cells create the complimentary strand, completing the double-stranded plasmid. At this point, the new DNA is integrated, and the recipient can now express any traits encoded on the plasmid, such as antibiotic resistance through the production of $\beta$-lactamases. Additionally, this transconjugant can now create a pilus and donate to other recipients. This process of transferring the genetic material of a plasmid is called transfection.

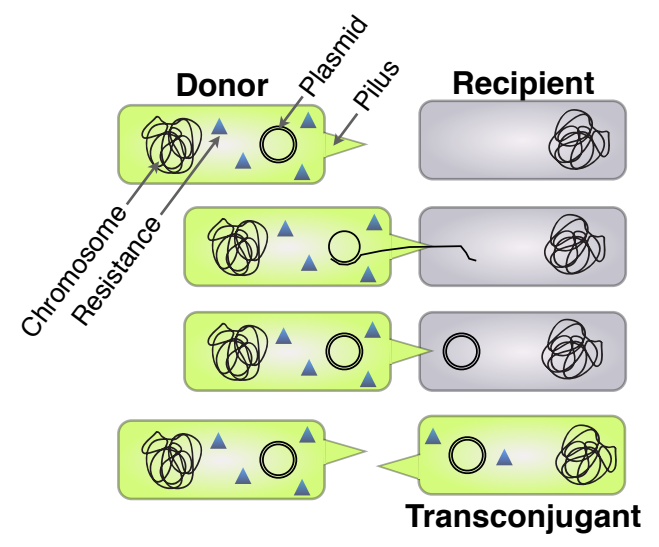

Figure 1: Illustration of plasmid transfer through bacterial conjugation. During this process, a donor (left) establishes direct contact and transfers a plasmid encoding antibiotic resistance to a recipient (right). This recipient can now exhibit resistance as well as donate the plasmid to others using its pilus.
Because horizontal gene transfer via plasmids is an integral process in bacterial life [4], understanding the environments under which this mechanism is favored is critical. Several mathematical models, notably those of Stewart and Levin, have been developed to study the evolutionary dynamics of bacterial plasmids, providing insights into the conditions that promote selection for mobile genetic elements in natural systems $[8,9,15]$. Most frequently, these models have been used to study the transfer of both conjugative and mobilizable plasmids. These studies have shown a wide range of conditions under which conjugationally-transmitted plasmids could become established, while the establishment of mobilizable plasmids was found to only occur in a small set of conditions. However, models including acquisition of plasmids through multiple conjugative events allowed for a wider range of conditions under which mobilizable plasmids could be established [13]. Recently, McGinty et al. examined the evolution of plasmid-encoded cooperative behaviors [11]. Here, they found that cooperation was not likely to be maintained in well-mixed populations, and that the battle between costly cooperators and non-cooperators occurred at the plasmid level. Aside from insights into the dynamics of populations in the presence of plasmids, these models have shown that parasitic plasmids, which provide little or no benefit, are likely to be rare [15].

Models such these are often based on differential equations, and as a result describe interactions of well-mixed populations in which the distribution of- and interaction among cells are random. Spatial relationships between individuals, however, have been shown to have significant effect on the dynamics of many types of interactions [2,5,17]. Spatial structure may be especially important in the case of conjugative plasmids, where cell-cell contact is required. For example, the dynamics of plasmid transfer in mice intestine were shown in one previous study to better resemble results in spatial rather than well-mixed settings [10]. In situations where the trait encoded on the plasmid in question produces a public benefit, such as the degradation of antibiotic modeled in this work, organisms must exhibit that behavior sufficiently to show a beneficial change globally before such costly behaviors are selected for. This is because the benefits provided are effectively distributed among all members of the population. In contrast, the benefits need only be observable on a local scale in spatially-structured populations, where benefits are distributed among neighbors only.

Spatial structure has recently begun to be incorporated into studies of plasmids $[6,14]$. Most similar to the work presented here, Krone et al. evolved populations on lattices, where growth rates among plasmid donors and recipients were tied to the availability of resource in the neighborhood [6]. The growth of cells and rates of plasmid transfer in that model were found to accurately match laboratory bacterial populations. That work also confirmed that initial cell densities greatly influenced the degree of plasmid transfer in spatially-structured populations, which differed from predictions arising from well-mixed models. Also in contrast to results from classic well-mixed models, the authors found that parasitic plasmids could in fact be maintained through high transfer rates. This result may help account for the abundance of so-called "cryptic" plasmids in nature, which provide no immediately apparent benefit to their carriers. The use of spatial structuring is continued in this work. 


\section{METHODS}

To study the roles that a plasmid's adaptive benefit and cost of carriage and conjugative transfer have on the composition of populations, we modeled a world consisting of host organisms and plasmids that those hosts could bear. Like their natural counterparts, plasmids potentially provided hosts with a beneficial trait. In our model, this behavior was the degradation of an antibiotic in the environment. Without degradation, this antibiotic was lethal to hosts, modeled as an increase in mortality rate. To allow for the possibility of non-beneficial plasmids, hosts could also be transfected with a plasmid that did not degrade antibiotic. Instead, its presence simply harmed its host. To combat these parasitic plasmids, hosts were able to evolve a costly behavior that prohibited transfection, thus preventing the reception of all plasmids.

To create this model, we used the a pre-release version of the SEEDS ${ }^{1}$ platform, a stochastic, asynchronous cellular automaton-like model based on graphs [2]. In this model, populations consisted of a set of 100000 cells, which were randomly placed on a plane. Each cell was then connected via an undirected edge to all other cells within a calculated distance. For this work, we used a distance that yielded an expected node degree of 20, meaning that each cell interacted with approximately this many neighbors. This model differs from previous models using lattices, where cells were connected to exactly 8 neighboring cells in which 0,1 , or 2 organisms resided [6]. Such differences in the number of interactions among cells have previously been shown to substantially affect the spread of behaviors [2].

Because naturally-occurring plasmids have no free state and depend on their host, each occupied cell in this world represented a host. At any point in time, each cell was in one of five possible states:

1. Free-Susceptible cells were not transconjugants of the plasmid, but remained susceptible to future transfection. As antibiotic resistance could only be provided by plasmids in this model, these cells were susceptible to being killed by the antibiotic.

2. Free-Resistant cells were not transconjugants of the plasmid and paid an additional cost to maintain resistance to transfection. These cells were also susceptible to being killed by the antibiotic.

3. Transconjugant-Sensitive cells bore a plasmid. However, this plasmid did not confer antibiotic resistance, leaving these cells susceptible to being killed by the antibiotic. These plasmids can be seen as parasites or "null" plasmids, which are costly to their host but do not provide any benefit [11].

4. Transconjugant-Insensitive cells bore the plasmid that conferred antibiotic resistance. These cells actively reduced the amount of antibiotic in their local environment, as described below.

5. Empty cells had no effect on their neighbors. When updated, an Empty cell adopted the strategy of a randomlyselected neighbor, further described below.

\footnotetext{
${ }^{1}$ https://github.com/briandconnelly/seeds
}

At the beginning of each experiment, cells were randomly assigned one of these five strategies. Each plasmid's rate of transfer was assigned a random value drawn from a uniform distribution between $\gamma_{\min }$ (0 in all experiments) and $\gamma_{\max }$. During each epoch, the unit of time in this model, 100000 cells were chosen at random and updated according to the rules described below. A state diagram highlighting these transitions is shown in Figure 2.

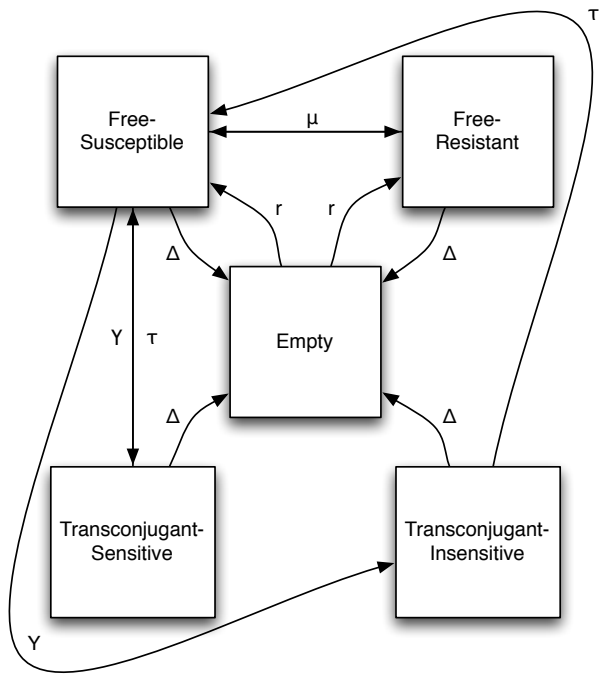

Figure 2: Available cell strategies and possible transitions among them. All strategies could transition to Empty through mortality $(\Delta)$. Empty cells could become either of the two Free strategies through replication $(\mathbf{r})$. Through mutations $(\mu)$ during replication, Free-Susceptible could become Free-Resistant and vice-versa. Free-Susceptible cells could become either Transconjugant-Sensitive or Transconjugant-Insensitive through conjugation $(\gamma)$, and Transconjugant-Sensitive or TransconjugantInsensitive could become Free-Susceptible through segregation $(\tau)$, the loss of plasmid. As vertical transmission was not modeled, there are no transitions from Empty cells to Transconjugant-Sensitive or Transconjugant-Insensitive cells.

Table 1 lists the values for all parameters to this model, described below. The growth of each strategy was controlled by its rate of mortality $(\Delta)$, which represented a cell's probability of dying during each update. All strategies shared an intrinsic death rate $\left(\Delta_{I}\right)$, and the costs associated with plasmid resistance $\left(C_{R}\right)$, plasmid carriage, and plasmid antibiotic resistance $\left(C_{A R}\right)$ manifested themselves as increases in death rate. This means that at any given time, a cell incurring higher costs through any of these mechanisms was more likely to die than cell without them. Transconjugants faced an increase in mortality directly associated with the evolved transfer rate of the plasmid $(\gamma)$, so frequent transfection of plasmids was costly. When a cell died through this process, it transitioned to Empty.

The death rate of a cell was also affected by the level of antibiotic in the surrounding cells. This antibiotic flowed into- and out of each cell at configured rates during each 
Table 1: Model Parameters Used in Experiments

\begin{tabular}{|c|c|c|}
\hline Parameter & Description & Value \\
\hline \hline$\Delta_{I}$ & Intrinsic death rate & 0.05 \\
$C_{r}$ & Cost of plasmid resistance & 0.05 \\
$C_{a r}$ & Cost of antibiotic resistance & 0.05 \\
$\gamma_{\min }$ & Minimum transfer rate & 0.0 \\
$\gamma_{\max }$ & Maximum transfer rate & $0.05-0.5$ \\
$a$ & Equilibrium per-cell antibiotic level & $0-9$ \\
$L D_{100}$ & $100 \%$ Lethal level of antibiotic & 10 \\
$d$ & Fraction of antibiotic degraded & 0.667 \\
$\tau$ & Segregation rate & 0.01 \\
$\mu$ & Mutation rate & 0.001 \\
$\mu_{\sigma}$ & Standard deviation of mutations & 0.01 \\
\hline
\end{tabular}

epoch, which if not degraded, reached an equilibrium level (a). As a cell updated, the mean level of antibiotic in that cell and the neighboring cells was calculated. The increase in mortality was then modeled as the fraction of this mean level over the configured $L D_{100}$, or level at which the antibiotic killed $100 \%$ of cells. Transconjugant-Insensitive cells, however, degraded a configured fraction $(d)$ of antibiotic in their cells as they were updated. Because mean neighborhood levels were used in calculating increases in mortality, a cell could survive in a neighborhood comprised of sufficient Transconjugant-Insensitive cells. In our experiments, $L D_{100}$ was kept at 10, and equilibrium per-cell levels of antibiotic between 0 and 9 were used. These settings resulted in an increase in mortality of between $0 \%$ and $90 \%$, respectively, in the absence of degradation.

The following equations describe the mortality rates of the Free-Susceptible (1), Free-Resistant (2), Transconjugant-Sensitive (3), and Transconjugant-Insensitive (4) strategies. Here, $G()$ captures the increase in mortality as related to the level of antibiotic in a cell's neighborhood.

$$
\begin{gathered}
\Delta_{F S}=\Delta_{I}+G() \\
\Delta_{F R}=\Delta_{I}+C_{r}+G() \\
\Delta_{T S}=\Delta_{I}+\gamma+G() \\
\Delta_{T I}=\Delta_{I}+\gamma+C_{a r}
\end{gathered}
$$

During each update, a cell could also change its strategy through the loss (segregation) or acquisition (conjugation) of the plasmid. Segregation was modeled as a separate death rate attributed to all plasmids $(\tau)$, and can be viewed as the vegetative segregation rate used in previous models [15]. The rate at which conjugation occurred depended on this evolved rate $(\gamma)$ of each plasmid, and the configured minimum $\left(\gamma_{\min }\right)$ and maximum $\left(\gamma_{\max }\right)$ rates. This relationship was modeled as a linear function, where a transfer rate equal to $\gamma_{\min }$ resulted in a $0 \%$ probability of being transferred at each epoch, and a transfer rate equal to $\gamma_{\max }$ resulted in a $100 \%$ probability of being transferred. Increases in a plasmid's transfer rate resulted in additional costs to the cell [19]. By changing the configured value of $\gamma_{\max }$, the cost of conjugative transfer was controlled. In other words, to achieve an $80 \%$ chance of transfer, $\gamma$ would have to be 0.2 if $\gamma_{\max }$ were 0.25 , or 0.4 if $\gamma_{\max }$ were 0.5 . The latter would therefore incur twice the mortality cost on the cell.
Conjugation was a density-dependent phenomenon, as it was successful only in the presence of Free cells. If horizontal transmission was attempted during the update of an Transconjugant cell, a random neighbor was chosen. If that neighbor was Free-Susceptible, it became transfected with the plasmid of the donor. During transfection, mutations altered the donor plasmid's rate of transfer with a configured probability $(\mu)$. This mutated transfer rate was drawn from a uniform distribution with mean equal to the rate of the donor plasmid and a configured standard deviation $\left(\mu_{\sigma}\right)$.

When an Empty cell was updated, it adopted the strategy of a randomly-selected neighboring cell. Because vertical transmission was not used in this work, plasmids were not transferred from plasmid-bearing parent cells. In this situation, the cell became Free-Susceptible.

Because a cell could replicate only into an adjacent Empty cell, space served as a limiting resource for hosts [16]. This limitation created a selective advantage for organisms that persisted long enough to spread to other cells. Also, in contrast to other computational models in which organisms replicate on top of others, killing the initial occupant in the process, Empty cells allowed death to be an explicit process rather than a byproduct arising from a cell eventually being overwritten by another organism. In this model, persistence could either be accomplished through a lowered mortality or by having resistance to the antibiotic. Which of these forces was stronger depended on the composition of the populations and the strength of the antibiotic treatment.

Although complex, this model makes several assumptions. The first is that plasmids can not be transferred vertically. Because plasmids can only spread and be maintained through horizontal transmission, evolved rates of transfer may be higher than expected as compensation. Previous work has demonstrated a tradeoff between vertical- and horizontal transmission [19]. Vertical transmission was omitted so that the role of HGT could be more directly examined. The second assumption is that transconjugants can carry only one plasmid. Therefore, any difference in dynamics introduced through the presence of multiple transfections is not captured in this model. Finally, as all plasmids in this model are able to be transferred to neighboring cells, this model captures only conjugative plasmids and excludes mobilizable plasmids.

This model was used to examine how both the level of antibiotic present in the environment (i.e., through different "doses") and the costs associated with transfer (through $\left.\gamma_{\max }\right)$ affect the prevalence of the plasmids in the system and the amount of resistance toward plasmids. For each environment, we examine the evolved level of transfer as well as the resulting abundances of each strategy. Although the costs of carrying a plasmid in our model depended directly on its rate of transfer, the benefits provided by that plasmid depended on both the level of antibiotic and the composition of the cells in the neighborhood. In other words, while a single transfected cell may not be have able to degrade the antibiotic enough to compensate for the costs incurred by bearing that plasmid, a group of neighboring Transconjugant cells may have.

For each experimental condition, five replicate populations were examined for 10000 epochs. Each population started with a different random seed, which yielded different outcomes for stochastic processes such as initial population composition, the distribution of transfer rates, cell update 


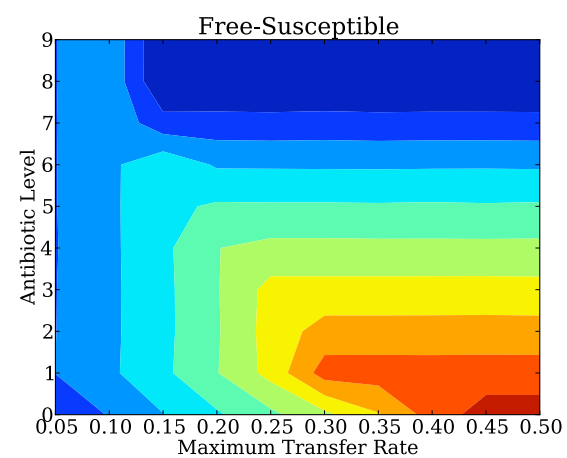

(a)

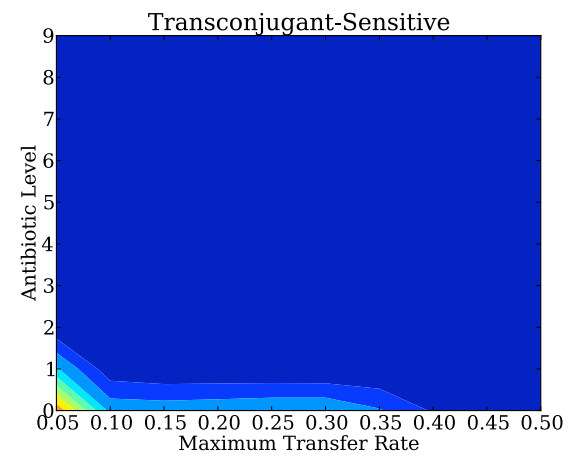

(c)

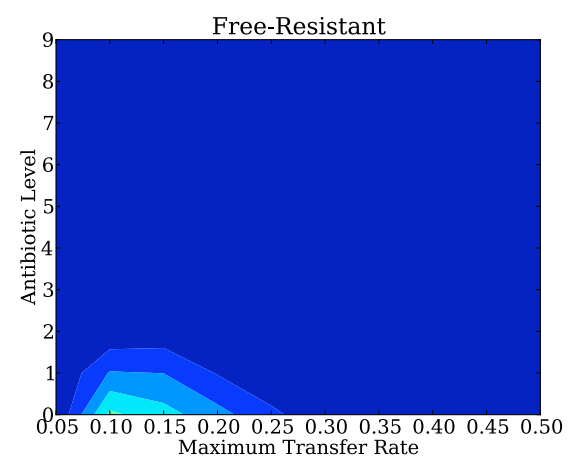

(b)

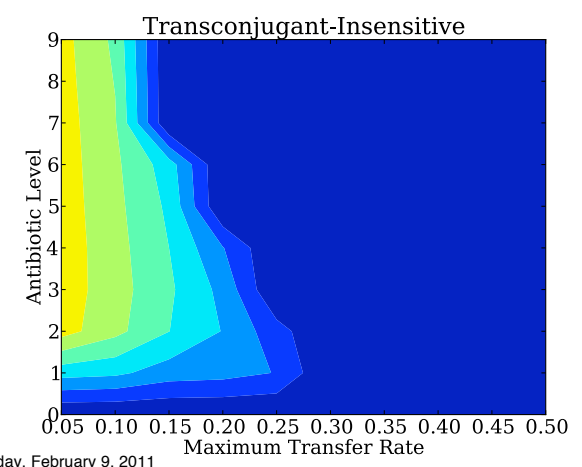

(d)

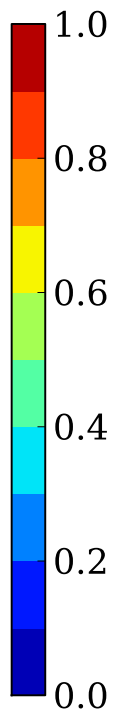

Figure 3: Mean fraction of cells occupied by each strategy after 10000 epochs. Scale is indicated on the right. (a) Free-Susceptible are able to reach substantial densities in most treatments. (b) In only a small set of parameters can Free-Resistant cells be maintained. This result is likely because the cost of resisting transconjugation outweighs the benefit. (c) Transconjugant-Sensitive cells are also rarely maintained. This result may contribute significantly to the rarity of the Free-Resistant strategy. (d) Transconjugant-Insensitive cells are maintained in a number of environments. Importantly, their maximum transfer rate (and therefore cost of bearing) is a larger determining factor to their maintainability than the level of antibiotic. Empty cells comprise roughly $\Delta_{I}$ percent of the populations.

order, neighbor selection, and mortality. The presence of such stochastic elements sets this and other similar models apart from mathematical models. This is distinction critical, as stochasticity has increasingly been shown to play a key role in evolution [7].

\section{RESULTS}

The proportion of each strategy in populations at the end of these experiments is shown in relation to configured maximum transfer rates and equilibrium levels of antibiotic in Figure 3. In these results, we consider strategies to be substantial when their densities were greater than that produced simply through drift, or 100 cells. This restriction corresponds with fractions of $0.1 \%$ in Figure 3.

As seen in Figure 3(a), the Free-Susceptible strategy was the most successful overall, which was likely attributable to its low mortality rate. Of course, these cells were susceptible to being killed by the antibiotic, so their success was greatest in environments with low levels of antibiotic. Interestingly, Free-Susceptible comprised the largest portion of the population in the low-antibiotic, high-maximum-transmissionrate environments. This result is likely because at these lev- els of $\gamma_{\max }$, frequent transfer of either plasmid type would be very costly to the host. In fact, neither of the plasmidbearing strategies was maintained in populations with $\gamma_{\max }$ values above 0.30 unless no antibiotic was present in the environment.

Resistance to plasmid transfection reached substantial densities in only a narrow range of conditions. This generally occurred when levels of antibiotic and $\gamma_{\max }$ were low. These conditions roughly coincided with those that supported substantial densities of Transconjugant-Sensitive cells (Figure $3(\mathrm{c})$ ). In these environments, selection for antibiotic resistance was low, and the relatively inexpensive cost of transfer allowed Transconjugant-Sensitive cells to persist. Hosts responded to this rise in parasitic plasmids by evolving resistance to plasmid transfection. This cost of resistance in turn caused evolved transfer rates in Transconjugant-Sensitive cells to decrease, as shown in Figure 4. This decrease in cost cost of Transconjugant-Sensitive cells enabled these plasmids to remain in the population in more environments.

As seen in Figure 3(d), the Transconjugant-Insensitive strategy was able persist in many of the environments examined. This is because these plasmids, although costly, provided benefit to their host. The benefit of this added be- 


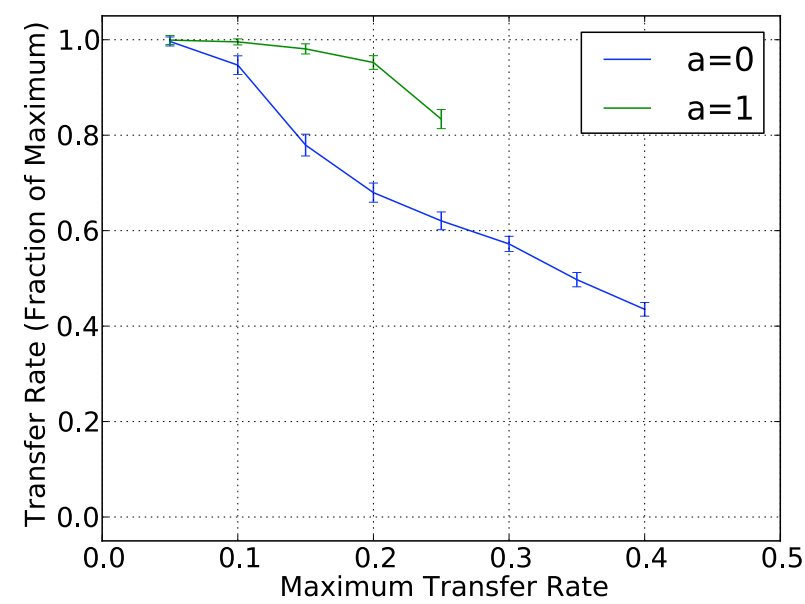

Figure 4: Evolved rates of transfer for Transconjugant-Sensitive cells in low-antibiotic environments. In order to persist despite costs to the hosts, these cells had to evolve restraint in their levels of transfer. Data are averaged over 5 replicates \pm SEM.

havior, which reduced the antibiotic-induced rates of mortality seen by cells sensitive to the antibiotic, was especially apparent in environments with high levels of antibiotic. In these environments, this strategy was dominant. Figure ?? shows the rates of transfer evolved in these populations in low $\gamma_{\max }$ environments. In the lower- $\gamma_{\max }$ environments where this strategy persisted, cells evolved maximum rates of transmission. As $\gamma_{\max }$ increased to 0.2 , some restraint appears to have emerged.

Because bearing the plasmid for antibiotic resistance was a costly behavior, this situation created selection for cells to take advantage of this public service. Therefore, in highantibiotic environments, both types of Free cell can be considered cheaters, which took advantage of the reduction of antibiotic performed by Transconjugant-Insensitive neighbors while not contributing themselves. This relationship can be seen in environments with per-cell equilibrium resource levels of 8 and 9 , where these are the only strategies that persist. As exemplified in Figure 6, when multiple strategies were maintained, clusters of strategies formed. These clusters constantly move as Free cells expand more quickly than Transconjugant-Insensitive cells. As these clusters of Free cells expand, however, they become more sensitive to the antibiotic, as there are fewer TransconjugantInsensitive cells to provide degradation. These cells are eventually killed, providing space for Transconjugant-Insensitive cells to grow, and this cycle continues.

\section{DISCUSSION}

This work has revealed the conditions under which beneficialand parasitic conjugative genetic elements can be maintained in a computational model. Specifically, as environmental conditions became more adverse through the introduction of an antibiotic, populations allowed for greater densities of plasmid-bearing cells as levels of the antibiotic increased.

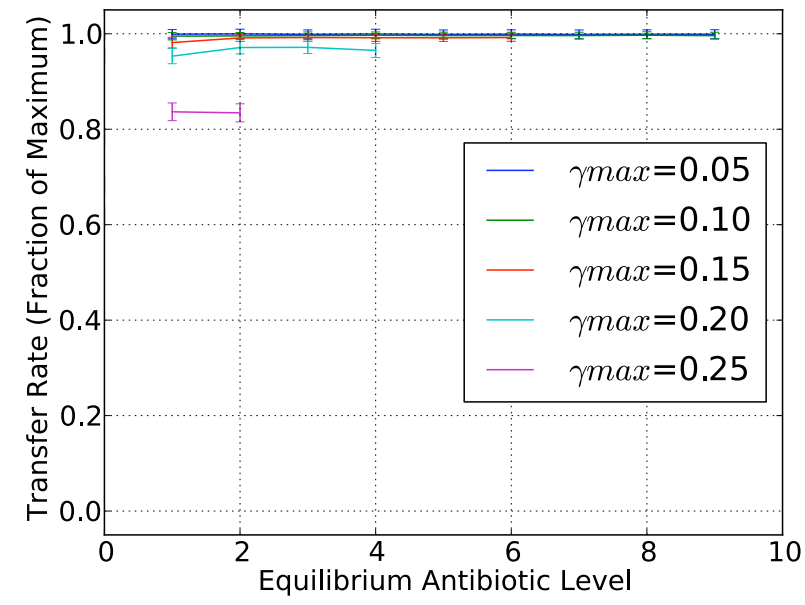

Figure 5: Evolved rates of transfer for Transconjugant-Insensitive cells in low- $\gamma_{\max }$ environments. Data are averaged over 5 replicates \pm SEM

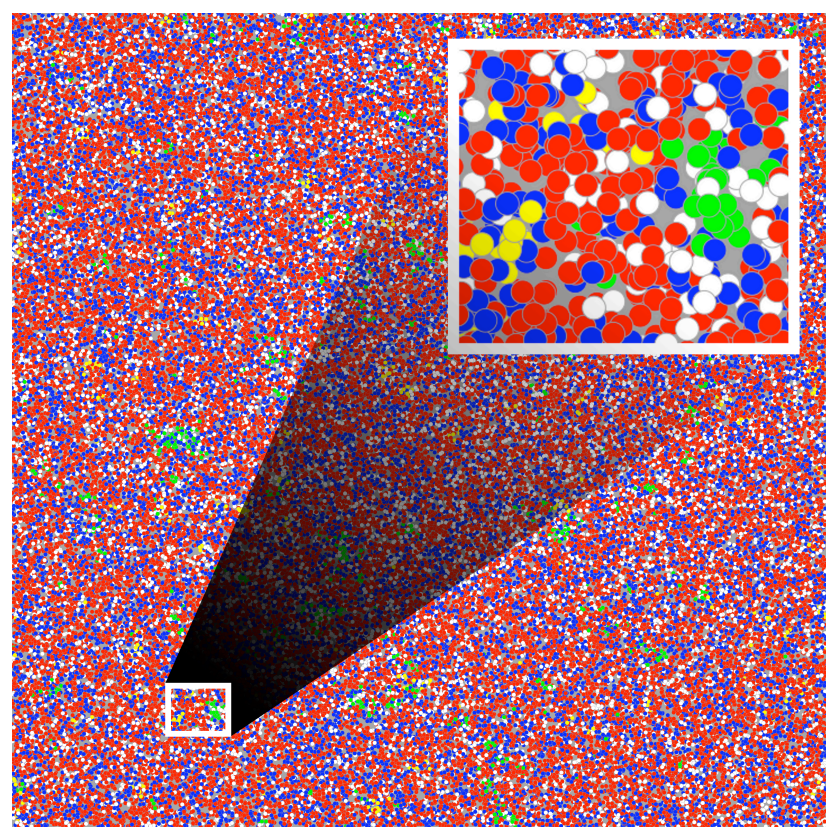

Figure 6: When multiple strategies coexisted in populations, those strategies formed clusters. In this case, Transconjugant-Insensitive (red) is the dominant strategy. While Free-Susceptible (blue), FreeResistant (green), and Transconjugant-Insensitive (yellow) maintain smaller clusters. 
This is because the benefit provided by plasmids bearing antibiotic resistance increased in these environments.

In certain situations, we also observed the maintenance of a parasitic plasmid, which was costly to transconjugants but did not confer any benefits. As a response, resistance to all plasmids emerged in these populations. We also observed the evolution of a slightly-restrained transmission rate in these plasmids as their costs approached the point at which they would otherwise become too costly to maintain.

Interestingly, populations rarely contained substantial densities of Free-Resistant cells, which were not involved in conjugation. This corresponds with the dearth of TransconjugantSensitive cells, which were parasitic to their host. Therefore, these environments provided little resistance to horizontal gene transfer.

As mentioned, the model presented in this work made several assumptions, each of which could alter the results. Several of the mechanisms not included will be incorporated into future models of this system. For example, allowing for vertical transmission may broaden the conditions under which plasmids could be maintained and provide a greater understanding of the tradeoff between the two methods [19]. Also, the possibility of hosts carrying multiple plasmids may present different dynamics. Previous work has indicated that this ability may expand the conditions under which plasmids can be maintained and possibly allow for mobilizable plasmids [13].

This work gives us a greater insight into how mechanisms of horizontal gene transfer such as conjugative plasmids can emerge and be maintained in populations of microorganisms. Because horizontal gene transfer plays a major role in many bacterial adaptations [18], models that capture its dynamics can be of great value from a medical standpoint. For example, models of bacterial processes such as quorum sensing [1] may shed light on the efficiency and long-term effects of new treatments that target these adaptations [3].

Through spatial models such as these, we can gain a greater understanding of the environments under which the process of horizontal gene transfer evolved and is maintained. Such knowledge may be important to understanding bacterial life and help to disentangle some of the branches on the tree of life.

\section{ACKNOWLEDGMENTS}

This work was supported in part by National Science Foundation grants CNS-0915855, CCF-0820220, and CNS-0751155; and by U.S. Army Grant W911NF-08-1-0495. Luis Zaman was supported by an AT\&T Labs Fellowship. This material is also based in part upon work supported by the National Science Foundation under Cooperative Agreement No. DBI-0939454. Any opinions, findings, and conclusions or recommendations expressed in this material are those of the authors and do not necessarily reflect the views of the National Science Foundation.

\section{REFERENCES}

[1] B.L. Bassler. Small talk: Cell-to-cell communication in bacteria. Cell, 109:421-424, 2002

[2] B. D. Connelly, L. Zaman, C. Ofria, and P.K. McKinley. Social structure and the maintenance of biodiversity. In Proceedings of the 12th International Conference on the Synthesis and Simulation of Living Systems (ALIFE), pages 461-468, 2010.

[3] T. Defoirdt, N. Boon, and P. Bossier. Can Bacteria Evolve Resistance to Quorum Sensing Disruption? PLoS Pathogens, 6(7):430-435, 2010.

[4] W. Doolittle. Uprooting the tree of life. Scientific American, 282(2):90-95, 2000.

[5] B. Kerr, M. Riley, M. Feldman, and B. Bohannan. Local dispersal promotes biodiversity in a real-life game of rock-paper-scissors. Nature, 418(6894):171-174, 2002.

[6] S. Krone, R. Lu, R. Fox, H. Suzuki, and E. Top. Modelling the spatial dynamics of plasmid transfer and persistence. Microbiology, 153(8):2803-2816, 2007.

[7] T. Lenormand, D. Roze, and F. Rousset. Stochasticity in evolution. Trends in Ecology 8 Evolution, 24(3):157-165, 2009.

[8] B. Levin and F. Stewart. The population biology of bacterial plasmids: a priori conditions for the existence of mobilizable nonconjugative factors. Genetics, 94(2):425-443, 1980.

[9] B. Levin, F. Stewart, and V. Rice. The kinetics of conjugative plasmid transmission: fit of a simple mass action model. Plasmid, 2(2):247-260, 1979.

[10] T. Licht, B. Christensen, K. Krogfelt, and S. Molin. Plasmid transfer in the animal intestine and other dynamic bacterial populations: the role of community structure and environment. Microbiology, 145(9):2615-2622, 1999.

[11] S.E. McGinty, D.J. Rankin, and S.P. Brown. Horizontal gene transfer and the evolution of bacterial cooperation. Evolution, 65(1):21-32, 2011.

[12] A. Norman, L.H. Hansen, and S.J. Sørensen. Conjugative plasmids: vessels of the communal gene pool. Philosophical Transactions of the Royal Society B: Biological Sciences, 364(1527):2275-2289, 2009.

[13] L. Simonsen. The existence conditions for bacterial plasmids: theory and reality. Microbial ecology, 22:187-205, 1991.

[14] F. Slater, K. Bruce, R. Ellis, A. Lilley, and S. Turner. Heterogeneous selection in a spatially structured environment affects fitness tradeoffs of plasmid carriage in pseudomonads. Applied and environmental microbiology, 74(10):3189-3197, 2008.

[15] F. Stewart and B. Levin. The population biology of bacterial plasmids: a priori conditions for the existence of conjugationally transmitted factors. Genetics, 87(2):209-228, 1977.

[16] D. Tilman. Resource competition and community structure. Princeton University Press, 1982.

[17] D. Tilman and P. Kareiva, editors. Spatial ecology: the role of space in population dynamics and interspecific interactions. Princeton University Press, 1997.

[18] E. Top, Y. Moënne-Loccoz, T. Pembroke, and C. Thomas. Phenotypic traits conferred by plasmids. In The Horizontal Gene Pool: Bacterial Plasmids and Gene Spread, pages 249-285. Harwood Academic Publishers, 2000.

[19] P. Turner, V. Cooper, and R. Lenski. Tradeoff between horizontal and vertical modes of transmission in bacterial plasmids. Evolution, 52(2):315-329, 1998. 